\title{
Cross-linking Peptide Vaccine Heat Shock Protein 72 and Alpha-fetoprotein Elicited Specific Dendritic Cells, Cytotoxic T-lymphocyte Cells and Natural Killing Cells
}

\author{
Xiao-Ping WANG ${ }^{1,{ }^{*}}$, Bing XU ${ }^{1}$, Huan-Ping $\mathrm{LIN}^{1}$, Qian $\mathrm{ZHAO}^{1}$, Ke-Pei ZHANG ${ }^{1}$, \\ Yi-Xin YANG ${ }^{2}$
}

${ }^{1}$ Key Laboratory of Molecular Biology and Pathology, Shaanxi University of Chinese Medicine, Xianyang, Shaanxi 712046, PR China

${ }^{2}$ Department of Biological Sciences, Emporia State University, Emporia 66801, Kansas USA

*wxpphd72@yahoo.com

${ }^{*}$ Corresponding author

Keywords: Alpha-fetoprotein (AFP), Heat Shock Protein 72 (HSP72), Cytotoxicity, Hepatocellular carcinoma $(\mathrm{HCC})$.

\begin{abstract}
Alpha-fetoprotein (AFP) is an oncofetal protein during Hepatocellular carcinoma (HCC) development which could generate weaker and less reproducible antitumor protection, and may serve as a target for immunotherapy. Therefore, it is imperative to enhance its immunogenicity and develop therapeutic vaccines to eliminate AFP-expressing tumors. In this study, by way of peptide synthesis method, we constructed a potential therapeutic peptide vaccine, heat shock protein 72 (HSP72) and AFP peptide containing the specific antigen epitope. Our results demonstrated that AFP peptide and HSP72 synergistically exhibited significant increases in AFP-specific dendritic cells, CD $8^{+} \mathrm{T}$ cells, natural killing cells responses and impressive antitumor effects against AFP-expressing tumors. Our study suggests that a tumor peptide vaccine by cross-linking AFP peptide and HSP72 peptide is a promising approach for HCC therapy.
\end{abstract}

\section{Introduction}

Heat shock proteins (HSP) have been verified to act as potent immunoadjuvant to enhance antigen-specific tumor immunity $[1,2]$. HSP play an important role, not only in the process of protein folding, transport and degradation, but also in participating in directing more efficient antigen presentation to $\mathrm{CD}^{+} \mathrm{T}$ cells through the MHC-I pathway [2]. This central role for HSP in therapeutic vaccine is facilitated by their dual functions in both chaperoning antigens and serving as an adjuvant. HSP-based tumor vaccine strategies have been highly successful in animal models and are undergoing testing in clinical trial [3-5]. Therefore, an innovative approach that combines with families of HSP will be likely to generate more potent antitumor effect.

$80 \%$ of Hepatocellular carcinoma (HCC) have a high expressing rate of alpha-fetoprotein (AFP), which may serve as a target for immunotherapy [6-8]. AFP is an oncofetal protein during HCC development which could generate weaker and less reproducible antitumor protection [9]. Therefore, a vaccine specifically targeting AFP is particularly desirable.

In the present study, we investigated whether the immunogenicity of AFP peptide containing antigen epitope FMNKFIYEI, could be improved by HSP72 molecules peptide 14-mer peptide TKDNNLLGRFELSG (TKD, aa 450-463) and whether HSP72 molecules peptide could synergistically enhance the potency of AFP peptide therapeutic effect and further evaluated the immune responses induced by HSP72/AFP peptide combined vaccine (HSP72-P/AFP-P).

\section{Material and Methods}

\section{Mice and Tumor Cell Lines}

Six- to eight-week-old female BALB/C mice were purchased from the Experimental Animal Center at Fourth Military Medical University. All animals were maintained under specific-pathogen-free 
conditions, and all procedures were performed according to approved protocols and in accordance with recommendations for the proper care of laboratory animals. The investigation was approved by the Ethics Committee on animal Study at Shaanxi University of Chinese Medicine (2004-4B). mAFP-producing $\mathrm{H} 22$ mice hepatocellular carcinoma cells were kindly provided by College of Biomedicine, Xi'an Jiaotong University.

\section{AFP Peptide, HSP72 Peptide and Conjugation}

Mouse Hsp72 protein, the N-terminal-extended 14-mer peptide TKDNNLLGRFELSG (TKD, aa 450-463) and AFP peptide containing epitope 158-166 amino acid, FMNKFIYEI, was synthesized from the ZiYuPeptides Co., Ltd (Shanghai, China). Lyophilized material was resuspended in sterile distilled water at $10 \mathrm{mg} / \mathrm{ml}$, aliquoted, and stored at $-70{ }^{\circ} \mathrm{C}$ until use. Protein concentration was determined using BCATM protein assay kit (Pierce Inc., Rockford, IL, USA). Aliquots of each conjugate were stored at $-70{ }^{\circ} \mathrm{C}$ until use.

\section{Mice Immunized with HSP72-P/AFP-P Complex}

Female BALB/C mice were randomly divided into HSP72-P/AFP-P group, AFP-P group, HSP72-P group, PBS control and empty group. Every group had 10 mice. Before injection, each group was diluted in saline to $100 \mu \mathrm{g} / 100 \mu \mathrm{l}$. Various groups of mice were injected into the left flank subcutaneously (s.c.). Priming and boosting was performed with 10 $\mathrm{g}$ HSP72-P/AFP-P, AFP-P or HSP72-P, whereas PBS was used as controls. Mice were boosted s.c. with above proteins twice at 2 weeks intervals after the first priming. Two weeks after last immunization, splenocytes were harvested and diluted to different concentrations.

\section{ELISPOT Assay}

The ELISPOT was used to measure the frequency of cells producing the cytokine IFN $-\gamma$ in splenocytes harvested from above immunized mice according to manufacturer's instruction. The color spots, representing cytokine producing cells, were counted using an ELISPOT Reader System.

\section{Granzyme B ELISA.}

1x106 splenocytes harvested from above immunized mice were added to the 96 well microplate along with $5 \mu \mathrm{g} / \mathrm{ml}$ of AFP peptide containing $10 \%$ fetal bovine serum, 10 units $/ \mathrm{ml}$ of mouse interleukin (IL)-2 (PEPRO Tech ET Ltd.). After cultured at $37{ }^{\circ} \mathrm{C}$ for $24 \mathrm{~h}$, granzyme B released by actived immune cells was measured using a mouse granzyme B ELISA kit (CSB-E08720m, Cusabio Biotech) according to manufacturer's instructions. Plates were counted on an ELISA reader at $490 \mathrm{~nm}$.

\section{Cytotoxic T-lymphocyte (CTL) Assays}

BALB/C mice were immunized subcutaneously (s.c.) as described above. Two weeks after the last boost, $2.5 \times 107$ splenocytes were cocultured with $5 \mu \mathrm{g} / \mathrm{ml}$ of AFP peptide containing $10 \%$ fetal bovine serum, 10 units/ml of mouse interleukin (IL)-2 in RPMI 1640 supplemented with $10 \% \mathrm{FCS}$ at $37{ }^{\circ} \mathrm{C}$ in $5 \% \mathrm{CO} 2$. After 5 days of stimulation, the viable splenocytes were recovered and used as effector cells, and the H22 cells or LLC cells were used as target cells. The Non-Radioactive Cytotoxicity Lactate Dehydrogenase (LDH) release assay Kit (Promega, 249 USA) was performed to measure the effector cells against $\mathrm{H} 22$ or LLC tumor cells in the ratios of 10:1, 20:1 and 40:1, according to the manufacturer's protocol. Specific lysis was calculated according to the formula: percent specific lysis $=[$ (experimental release value - effector spontaneous release value - target spontaneous release value)/(target maximum release value - target spontaneous release value) $] \times 100$. Results shown are representative of experiments repeated three times.

\section{Dendritic Cell, CD8+ T cell and Natural Killing Cell Depletion CTL Assays}

To test dendritic cell, CD8+ T cell or natural killing cell response, a separate set of immunized mice were sacrificed and spleens were collected. Dendritic cells, CD8+ T cells or natural killing cells were depleted from spleen suspensions with anti-CD11+, anti-CD8+ or anti-NK Micro-Beads. The remaining splenocytes were cocultured with $5 \mu \mathrm{g} / \mathrm{ml}$ of AFP peptide containing $10 \%$ fetal bovine 
serum, 10 units/ml of mouse interleukin (IL)-2 in RPMI 1640 supplemented with $10 \% \mathrm{FCS}$ at $37{ }^{\circ} \mathrm{C}$ in $5 \% \mathrm{CO} 2$. After 5 days of stimulation, the splenocytes were used as effector cells and co-cultured with the $\mathrm{H} 22$ cells as described above.

\section{In Vivo Tumor Therapeutic Experiments}

To test the ability of HSP72-P/AFP-P vaccination to inhibit the growth of established tumors, $\mathrm{BALB} / \mathrm{C}$ mice in every group were injected subcutaneously in the left flank with $5 \times 105 \mathrm{H} 22$ cells per mouse on day 0 , and then injected subcutaneously in the right flank with different vaccination treatments on day 3,10, and 17 as above described. H22 tumor cells were washed after enzymatic digestion and resuspended in $0.2 \mathrm{ml}$ of PBS per animal, then injected s.c. into the left flank, while PBS was used as control. The tumor growth was monitored every day. Tumor size was measured in two dimensions with calipers every 3 days one week after tumor inoculation. Tumor volume was calculated using the formula: $\mathrm{V}=(\mathrm{a} 2 \mathrm{~b}) / 2$. Percentage of tumor-free mice was recorded and the survival of mice was monitored for eight weeks from the day of tumor challenge.

\section{Statistical Analysis}

All data expressed as means \pm S.D. Comparisons between individual data points were made using a Student's t-test. The frequencies of IFN- $\gamma$-producing splenic cells were valued using $\chi 2$ test. The Student's t test was performed to analyze the significance of differences between final tumor volumes of different groups of animals. $\mathrm{P}<0.05$ was considered statistically significant.

\section{Results and Discussion}

\section{Prime-boost Vaccines Generate AFP-specific Immune T Cells in Vivo}

Two weeks after the last immunization, splenocytes were harvested and stimulated with AFP peptide. Immunization of BALB/C mice with HSP72-P/AFP-P vaccine elicited much more strong T-cells responses than those with AFP-P immunization, whereas a vaccination with HSP72-P or AFP-P alone produced a small one. $640 \mathrm{IFN}-\gamma$ spot-forming immune $\mathrm{T}$ cells specific for AFP protein were detected per 1x106 splenocytes derived from the HSP72-P/AFP-P vaccinated mice, compared with only 15 of those derived from AFP-P vaccinated mice. Therefore, the numbers of IFN- $\gamma$-producing immune $\mathrm{T}$ cells in the splenocytes from mice immunized with HSP72-P/AFP-P were 30 times more than those from mice immunized with the HSP72-P or those from mice vaccinated with AFP-P $(\mathrm{P}<0.01)$. Our data suggested that HSP72-P/AFP-P vaccine generated significantly higher AFP-specific immune $T$ cells response compared with the other vaccinated groups.

HSP72-P/AFP-P immunized mice produced much higher concentration of granzyme B than those immunized with AFP-P, HSP72-P and PBS groups. The concentration of granzyme B in the splenocytes from mice immunized with HSP72-P/AFP-P were 15 times more than those from mice immunized with the HSP72-P or those from mice vaccinated with AFP-P $(\mathrm{P}<0.01)$. The results indicated that HSP72-P/AFP-P vaccine induced significantly higher AFP-specific immune T cells response compared with the other vaccinated groups.

\section{HSP72-P/AFP-P Significantly Primes Potent AFP-specific DC cells, CD8+ T Cell and Natural Killing Cell Responses}

Effector cells from mice immunized with HSP72-P/AFP-P showed much more stronger cytolytic effects on $\mathrm{H} 22$ target cells than those from mice vaccinated with HSP72-P, AFP-P or PBS $(\mathrm{P}<0.01)$. In addition, this cytolytic activity was specific for $\mathrm{H} 22$ cells, not for LLC cells $(\mathrm{P}<0.01)$. Our results also indicated that the cross linkage of AFP peptide to HSP72-P was required for enhancement of specific immune T cell activity, since the administration of AFP-P or HSP72-P only exhibited lower cytolytic activity. In contrast, splenocytes from the PBS control and empty group showed much lower levels of lysis of target cells.

To elucidate whether DC cells, CD8+ T cells or natural killing cells participate in the cytotoxic against $\mathrm{H} 22$ tumor cells, DC cell, CD8+ T cell and natural killing cell depletion assay were determined. 
After DC cells, CD8+ T cells or natural killing cells depletion, the remaining splenocyte effector cells from mice vaccinated with HSP72-P/AFP-P showed lower cytolytic effects on H22 target cells, especially for CD8+ T cells depletion. While DC cell, CD8+ T cell and natural killing cell were depleted, the remaining splenocytes demonstrated a much more lower cytolytic effects on target cells. There was no significant difference from mice treated by HSP72-P, AFP-P or PBS alone (P > 0.05). Our results indicated that DC cells, CD8+ T cells and natural killing cells concurrently participated in the specific cytolytic activity against H22 tumor cells via HSP72-P/AFP-P immunization.

\section{HSP72-P/AFP-P Vaccination Induce Therapeutic Immunity Against $\mathbf{H 2 2}$ Tumors}

The growth of tumor was monitored for about 4 weeks since day 7 post tumor inoculation. $100 \%$ of the mice immunized with HSP72-P/AFP-P had tumor-free survival for more than 60 days. In contrast, the mice receiving HSP72-P or AFP-P developed tumor growth within 10 days after tumor challenge, indicating that cross linkage between AFP-P and HSP72-P is essential for effective therapy. Mice immunized with HSP72-P or AFP-P alone demonstrated the higher average tumor volumes than mice immunized with HSP72-P/AFP-P ( $<<0.05)$. There was a statistically significant difference in the therapeutic effect of $\mathrm{H} 22$ tumor between HSP72-P/AFP-P and AFP-P vaccinated group (P < 0.01$)$. However, there was no statistically difference in the tumor mass among the groups immunized with AFP-P, HSP72-P and the group administrated with PBS. Different vaccinations greatly influenced the survival of mice. The mice immunized with AFP-P or HSP72-P all died before day 49 and all the mice immunized with HSP72-P/AFP-P still survived at day 60, whereas all the mice treated with PBS demised before day 35. These data suggested that HSP72-P/AFP-P immunization could significantly reduce tumor size and prolong the survival time of the tumor-bearing mice than AFP-P immunization alone, indicating that the vaccination of HSP72-P/AFP-P induced a desirable therapeutic effect against $\mathrm{H} 22$ tumor cells loaded in vivo. These results showed that vaccination with HSP72-P/AFP-P could eradicate previously inoculated AFP-expressing tumors in mice and inhibit the growth of tumors, inducing a stronger anti-tumor activity in vivo.

Recent studies on the immunodominant epitopes of AFP have provided a solution to the obstacle of HCC immunotherapy. AFP is produced at low serum levels after birth throughout life. The majority of human HCC overexpress the oncofetal antigen AFP, Mr 64,000 72,000 glycoprotein. Despite being exposed to high plasma levels of this oncofetal protein during embryonic development, body still displays a low immunity to it [10]. Butterfield has found that four peptides of human AFP processed and presented in the context of HLA-A0201, could be recognized by the human T cell repertoire, and could be used to generate AFP-specific CTL in human T cell cultures [11]. It was also found that murine immune system could generate $\mathrm{T}$-cell responses to this oncofetal antigen. Therefore, it may be an ideal target for immunotherapy. But AFP immunization alone still results in lower levels of specific response and poorly reproducible protective immunity $[6,9]$.

How to enhance host's active immunity to AFP may be an interesting strategy for HCC therapy.In this study, we developed a strategy to construct an innovative protein vaccine. Conjugation of HSP72-P and AFP-P with lower concentration of glutaraldehyde not only established the HSP72-P/AFP-P vaccine but also preserved the activity of HSP72-P immunoadjuvant and immunogenity of AFP peptide antigen, which was confirmed by the strong ex vivo and in vivo anti-tumor effect. We attributed the successful alpha-fetoprotein specific T-cell responses in mice to HSP72-P through mediating APCs to efficiently take up and process AFP peptide. Researches have shown that HSP72 itself has no antigenicity and its immunogenicity has been attributed to the peptides chaperoned by HSP72 [12]. It has been verified that HSP72 was a better molecule chaperon and adjuvant which could process and present weak tumor antigen to MHC-I of host APCs, eliciting specific T-cell response and CTL reaction $[13,14]$.

\section{Summary}

Our results indicate that HSP72-P/AFP-P vaccine can generate an impressive antitumor effect against AFP-expressing murine tumors through enhancement of AFP-specific DC cell, $\mathrm{CD}^{+} \mathrm{T}$ cell and natural killing cell mediated immune responses. Therefore, the tumor vaccine by cross-linking tumor 
antigen peptide and HSP72 peptide is a promising approach for cancer therapy which may be potentially applied to other cancer systems with known tumor-specific antigens.

\section{Acknowledgements}

This work is supported by Scientific Research Program Funded by Shaanxi Provincial Education Department (No.2007JK233, 14JS025), the Key Project of Ministry of Education of China (No.205002) and the National Natural Science Foundation of China (No.81172135, 81310108008).

\section{References}

[1] H. Singh-Jasuja, N. Hilf, H.U. Scherer, D. Arnold-Schild, H.G. Rammensee, R.E. Toes, et al: The heat shock protein gp96: a receptor-targeted cross-priming carrier and activator of dendritic cells. Cell Stress Chaperones Vol. 5 (2000), p. 462.

[2] W.K. Balker, J. Keeble and C. Colaco: Mycobacterial heat shock proteins as vaccines: a model of facilitated antigen presentation. Curr. Mol. Med. Vol. 7 (2007), p. 339.

[3] X.P. Wang, G.Z. Liu, A.L. Song, H.Y. Li, Y. Liu: Antitumor immunity induced by DNA vaccine encoding alpha-fetoprotein/heat shock protein 70. World J. Gastroenterol. Vol. 10 (2004), p. 3197.

[4] B. Liu, D. Ye, X. Song, X. Zhao, L. Yi, J. Song, et al: A novel therapeutic fusion protein vaccine by two different families of heat shock proteins linked with HPV16 E7 generates potent antitumor immunity and antiangiogenesis. Vaccine Vol. 26 (2008), p. 1387.

[5] Y.H. Lan, Y.G. Li, Z.W. Liang, M. Chen, M.L. Peng, L. Tang, et al: A DNA vaccine against chimeric AFP enhanced by HSP70 suppresses growth of hepatocellular carcinoma. Cancer Immunol. Immunother. Vol. 56 (2007), p. 1009.

[6] P. Hanke, C. Rabe, M. Serwe, S. Böhm, C. Pagenstecher, T. Sauerbruch, et al: Cirrhotic patients with or without hepatocellular carcinoma harbour AFP-specific T-lymphocytes that can be activated in vitro by human alpha-fetoprotein. Scand. J. Gastroenterol. Vol. 37 (2002), p. 949 .

[7] J.A. Marrero and K.S. Henley: The role of serum biomarkers in hepatocellular carcinoma surveillance. Gastroenterol. Hepatol (NY). Vol. 7 (2011), p. 821.

[8] J.H. Zhong, H. Li, L.Q. Li, X.M. You, Y. Zhang, Y.N. Zhao, et al: Adjuvant therapy options following curative treatment of hepatocellular carcinoma: A systematic review of randomized trials. Eur. J. Surg. Oncol. Vol. 38 (2012), p. 286.

[9] A. Saeki, K. Nakao, Y. Nagayama, K. Yanagi, K. Matsumoto, T. Hayashi, et al: Diverse efficacy of vaccination therapy using the alpha-fetoprotein gene against mouse hepatocellular carcinoma. Int. J. Mol. Med. Vol. 13 (2004), p. 111.

[10] H.F. Deutsch: Chemistry and biology of a-fetoprotein. Adv. Cancer Res. Vol. 56 (1991), p. 253

[11] L.H. Butterfield, A. Koh, W. Meng, C.M. Vollmer, A. Ribas, V. Dissette, et al: Generation of human T-cell responses to an HLA-A2.1-restricted peptide epitope derived from alpha-fetoprotein. Cancer Res. Vol. 59 (1999), p. 3134.

[12] J. Gong, Y. Zhang, J. Durfee, D. Weng, C. Liu, S. Koido, et al: A heat shock protein 70-based vaccine with enhanced immunogenicity for clinical use. J. Immunol. Vol. 184 (2010), p. 488.

[13] A.L. Myers, J.A. Krewet and M.R. Shah: Tumor immunity and prolonged survival following combined adenovirus-HSP72 and CEA-plasmid vaccination. Vaccine Vol. 23 (2005), p. 3565.

[14] F. Chalmin, S. Ladoire, G. Mignot, J. Vincent, M. Bruchard, J.P. Remy-Martin, et al: Membrane-associated Hsp72 from tumor-derived exosomes mediates STAT3-dependent immunosuppressive function of mouse and human myeloid-derived suppressor cells. J. Clin. Invest. Vol. 120 (2010), p. 457. 